ELOHI

Peuples indigènes et environnement

5-6| 2014

Ressources du vivant

\title{
Entomoterapia: curaciones entre los antiguos pueblos mayas de la península de Yucatán, México
}

Diana Cahuich-Campos y Fabio Flores Granados

\section{OpenEdition}

1 Journals

Edición electrónica

URL: http://journals.openedition.org/elohi/712

DOI: $10.4000 /$ elohi.712

ISSN: 2268-5243

Editor

Presses universitaires de Bordeaux

\section{Edición impresa}

Fecha de publicación: 1 enero 2014

Paginación: 39-54

ISBN: 979-10-300-0021-4

ISSN: 2431-8175

Referencia electrónica

Diana Cahuich-Campos y Fabio Flores Granados, « Entomoterapia: curaciones entre los antiguos pueblos mayas de la península de Yucatán, México », ELOHI [En línea], 5-6 | 2014, Publicado el 01 enero 2015, consultado el 12 noviembre 2019. URL : http://journals.openedition.org/elohi/712 ; DOI : 10.4000/elohi.712

\section{(c) PUB-CLIMAS}




\title{
Entomoterapia: curaciones entre los antiguos pueblos mayas de la península de Yucatán, México
}

\author{
DIANA CAHUICH-CAMPOSY FABIO FLORES GRANADOS \\ Universidad Nacional Autónoma de México
}

\section{Introducción}

Desde la antigüedad el uso de distintos tipos de artrópodos, particularmente insectos y los productos extraídos de estos animales, han sido parte de los recursos terapéuticos en los sistemas médicos de muchas culturas alrededor del mundo e incluso han tenido un papel místico y mágico en el tratamiento de muy diversas enfermedades (Costa-Neto 2005; Costa-Neto et al. 2006). Aunque relativamente simple, tanto la anatomía, fisiología y comportamiento, como el papel ecológico y desarrollo evolutivo de los artrópodos les permitiría adaptarse a prácticamente todos los ambientes acuáticos y terrestres, siendo los insectos el grupo taxonómico más diverso y numeroso, ya que representa alrededor del $85 \%$ de toda la fauna en el planeta, lo que equivale al $65 \%$ de toda la diversidad de especies hasta ahora conocidas (Brusca y Brusca: 593-600). Si bien una buena cantidad de insectos y arácnidos han sido ampliamente estudiados por actuar como parásitos, alergizantes, ponzoñosos, o vectores de diversas enfermedades en humanos, animales y plantas (De la Fuente) el conocimiento de sus propiedades curativas, así como del uso medicinal que a estos les dieran las antiguas culturas, es un campo aún poco explorado.

Hoy día, la utilización de casi 60 especies de insectos entre los grupos indígenas de Latinoamérica, cuyas propiedades inmunológicas, analgésicas, antibacteriales, diuréticas, anestésicas y anti-reumáticas han sido constatadas por 
la ciencia médica (Alves y Alves), sustenta la idea de que no pocos insectos y otros artrópodos fueron ampliamente utilizados en el pasado y que, dadas sus propiedades curativas, dicha tradición perdura desde mucho tiempo antes de la época prehispánica en Mesoamérica (Ramos-Elorduy y Pino: 195-196). Para el mismo territorio contamos con registros de, al menos, 252 especies que los pueblos zapotecos, mixtecos y mayas, entre muchos otros, siguen empleando en la actualidad para aliviar diversas enfermedades y curar padecimientos digestivos, respiratorios, nerviosos, circulatorios y óseos, involucrando para ello la utilización de diversas partes y productos de los mismos animales con los que, a su vez, se elaboran una variedad de sustancias y remedios terapéuticos (Ramos-Elorduy y Pino, ídem).

Dado que al alimentarse, los insectos, arácnidos, miriópodos y crustáceos asimilan muy diversas sustancias químicas, que luego transforman en compuestos bioactivos concentrados con propiedades específicas, los ancestrales remedios indígenas encierran, en sí mismos, diversos principios homeopáticos y naturistas (Serrano-Gonzalez et al.: 24-25). Ello explica que en la medicina tradicional mesoamericana, al igual que en la de muchas otras culturas del continente americano, dichos artrópodos, sean solos o mezclados, enteros, molidos, en emplastos, infusiones o tostados, han sido empleados desde el pasado para curar afecciones respiratorias, renales, hepáticas, estomacales, intestinales, parasitarias, pulmonares, bronquiales, pancreáticas, del aparato reproductor, renales, dermatológicas, endocrinas e inmunológicas, entre otras. (Cahuich-Campos: 16-17).

\section{Entomoterapia del área maya peninsular}

Perdido su origen en el pasado, las antiguas nociones entomológicas fueron registradas en diversas fuentes coloniales tempranas y su transmisión, de generación en generación, no solo habla de la vigencia de aquellos conocimientos sino también avala su eficacia a través de las prácticas de sanación que no pocos curanderos del área maya continúan ejerciendo (Flores 2014).

Desde la época precolombina y al igual que muy diversos animales, los artrópodos tuvieron gran importancia para los habitantes del área cultural mesoamericana, así como para muchos otros pueblos cuyo desarrollo tuvo lugar más allá de sus fronteras septentrional y meridional (Serrano-González et al:: 24-26). Ya fuese por sus propiedades curativas, o por determinadas características biológicas, no pocos fueron los pueblos que generaron, y aún preservan, profundos conocimientos sobre estos organismos, tanto por ser parte de la dieta alimenticia o para otros usos, como por ser la base de distintas prácticas 
terapéuticas, o incluso por ser considerados como animales agoreros y marcadores ambientales (Flores 2014) (fig. 1).

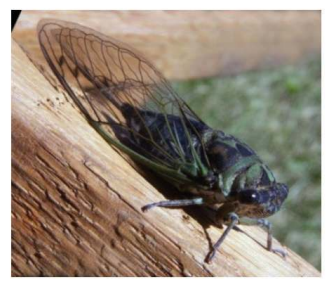

Chicharra (Cicadidae) / Xikitin / agorero de que la lluvia continuará

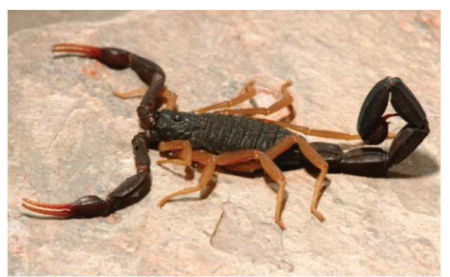

Alacrán (Centuroides sp.) / Síina'an / marcador climático del inicio del estiaje

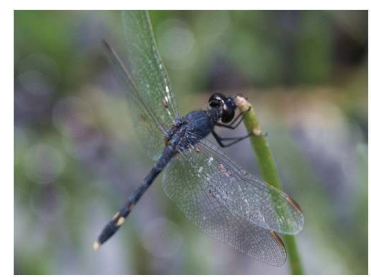

Libélula

(Anisoptera) / Tulix / agorero de que habrá visitas en casa

Figura 1. Insectos y arácnido comunes en el área maya peninsular con distintas etnocategorías de uso (Flores 2014) ${ }^{1}$

Así, ante la falta de estudios arqueológicos enfocados en las antiguas formas de curar enfermedades, las fuentes coloniales tempranas constituyen una alternativa de aproximación, puesto que algunos de dichos documentos guardan valiosa información sobre las nociones naturalistas que los pueblos mesoamericanos, al igual que muchos otros, desarrollaron mucho tiempo antes de la Conquista. Ejemplos de ello son el Códice Florentino, el Libellus de Medicinalibus Indorum Herbis, y la Historia Natural de Nueva España, que versan sobre los conocimientos naturalistas de pueblos nahuas del centro de México, quienes utilizaron y clasificaron muy distintos artrópodos útiles, y en los que se reportan al menos 14 de estos animales (pertenecientes a nueve órdenes), que curan 35 enfermedades (Serrano-González et. al.).

Junto con diversa información histórica (Hart; Peniche; Bracamonte y Sosa) y el sustento de datos etnográficos compilados en campo (Flores 2014) el examen minucioso de dichas fuentes cobra particular relevancia, no solo para lograr recuperar aquellos saberes ancestrales sino también para conocer las distintas formas de aprovechamiento de los recursos faunísticos y vegetales involucrados en los procesos de salud y enfermedad de los pueblos mesoamericanos del pasado.

\section{El contexto natural y cultural}

El extenso y heterogéneo territorio que suele conceptuarse como el área maya, con alrededor de $400,000 \mathrm{Km}^{2}$, es escenario de una notable diversidad cultural

1. Registros de trabajo de campo aún no publicados. Distintos informantes mayahablantes. 
manifiesta en más de veinte grupos lingüísticos compuestos en su mayoría de población indígena cuyas prácticas de aprovechamiento de la biota, desde las tierras altas a las tierras bajas y zonas costeras, han sido estrategias ampliamente difundidas y de gran profundidad histórica (Pérez Suárez).

Sin embargo, a pesar de que en muchas comunidades persisten muy diversas estrategias tradicionales de apropiación, y aunque los conocimientos etnobiológicos del área maya peninsular son los mejor documentados entre las lenguas mesoamericanas (Flores 2014), los datos concernientes a las relaciones humanos/artrópodos siguen siendo muy escasos, destacando la falta de estudios sobre la visión que los pueblos mayas tienen, y han tenido, de este tipo de animales. A diferencia de los no pocos ejemplos de aquellas plantas y animales significativos en la época prehispánica por su valor simbólico, económico o ecológico (Marcus; Schlesinger; Schele y Freidel, entre otros), la información sobre los artrópodos en general, así como su utilización específica en diversos tratamientos terapéuticos, y las enfermedades que eran tratadas con estos animales, son datos prácticamente inexistentes.

$\mathrm{Al}$ igual de como pudo suceder en otros lugares de Mesoamérica, las actuales prácticas de medicina tradicional en la península de Yucatán se remontan a la época precolombina, y de ello dan amplia cuenta fuentes etnohistóricas como Landa y Herrera y Tordesillas, en las que se señala que desde el pasado, existían en la región sacerdotes médicos o especialistas en el arte de curar, los que además utilizaban ampliamente muy distintas plantas, hongos y animales con propiedades medicinales. En este sentido, los ensalmos y conjuros descritos en el Ritual de los Bacabes (Arzápalo), copia de un códice del siglo VII, y cuya escritura, a finales del siglo $\mathrm{XVI}^{2}$, representan ventanas para asomarse al antiguo concepto maya de la enfermedad así como a distintas creencias sobre su origen y el papel que en ello jugaron los médicos ritualistas o $h$-men, "el que sabe". ${ }^{3}$ Fortuitamente, en algunas de aquellas encantaciones, traducidas del maya por escribas indígenas, afloran muy diversos conceptos y saberes relacionados con las antiguas prácticas de entomoterapia dado que la obra no solo contiene remedios para los piquetes de avispas o incluso tratamientos para afecciones renales usando artrópodos como las cochinillas ${ }^{4}$ sino también porque incluye imprecaciones para tratar el encantamiento de las arañas o por ejemplo, para cuando una persona aparecía en la puerta del $h$-men con una picadura de alacrán (Centuroides sp.), para que este lo conjurase.

2. Transcripto en caracteres latinos, y encontrado en Nunkiki, Yucatán, México.

3. Especialistas rituales mayas, terapeutas, yerbateros, hechiceros, "el que sabe".

4. Filo Artrópoda, subfilo Crustáceo, clase Malacostraca, orden Isópoda, suborden Oniscidea. 


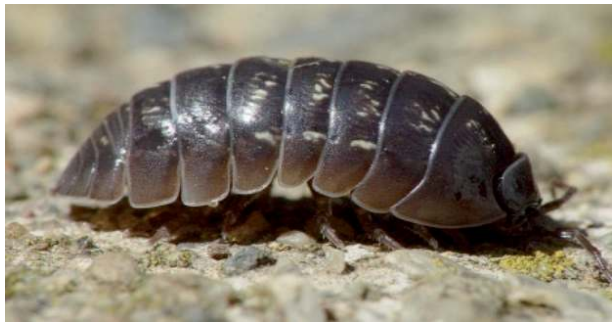

Cochinilla (Isópoda, suborden Oniscidea)

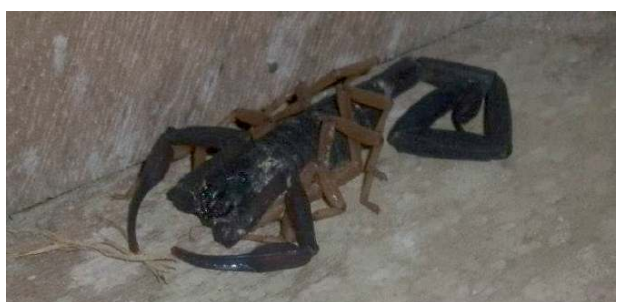

Alacrán negro (Centuroides gracilis Latreille)

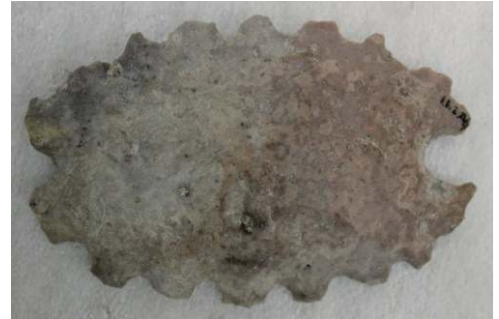

Excéntrico de sílex en forma de cochinilla (i?), cortesía del Museo Popol Vuh, Guatemala

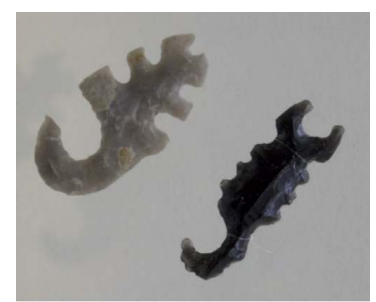

Excéntricos de sílex y obsidiana en forma de alacranes, cortesía del Museo Popol Vuh, Guatemala

Figura 2. Artrópodos del área maya, y artefactos líticos asociados a contextos rituales y ofrendas del Clásico tardío hallados en la Plaza Mayor de Dos Pilas en el Petén Guatemalteco (Stiver 1993, citado por Flores 2014).

De igual forma, en el mismo texto emergen diversas estampas o descripciones que permiten recrear determinadas prácticas de curación en las que dichos especialistas apelaban a los dioses para que sus remedios, que operaban tanto a nivel simbólico como pragmático, tuviesen los efectos deseados en la persona enferma o también para cuando el $h$-men "luchaba" con los espíritus-vientos que, como causantes de la enfermedad, tenían necesariamente que ser conjurados. Enmarcados en la antigua perspectiva cíclica mesoamericana que implicaba en todo momento sucesivos eventos de destrucción y creación del tiempo y en los que se veían inmersos la vida y la muerte, este tipo de pasajes ilustran la eventual necesidad de aquellos especialistas no solo de conocer las artes médicas sino también de tener las cualidades necesarias, los saberes y el poder, para lograr mediar con las fuerzas sobrenaturales la ocurrencia de la enfermedad misma. Echando mano de muy diversas suertes de prognosis o adivinación dirigidas a evitar la muerte de las personas afectadas por algún padecimiento cuyo tratamiento involucrase el uso de algún artrópodo, la curación o terapéutica empleada debió fundamentarse no solo en profundos conocimientos entomológicos por parte de los médicos ritualistas sino también en la capacidad de observar muy diversos signos naturales que regían el mismo acto de sanación. Así, no es fortuito que la acción de adivinar, registrada en el norte peninsular 
como k'intah o kinyah (Barrera-Vázquez 1991: 403-04; Pérez: 177), aluda tanto al sacerdote de ídolos como a la acción específica de medicar con augurios, hechizos y rogativas. En este sentido, la información epigráfica sustenta la idea de que tales nociones pudieron ser construidas en la época prehispánica, cuando los sabios o chilames invocaban a alguna divinidad o fuerza sobrenatural para que esta interviniese, evitara o propiciara, la ocurrencia de determinado padecimiento o enfermedad en las personas (fig. 2).

Por otra parte, diccionarios maya-españoles, como el Calepino de Motul (Ciudad Real), el Bocabulario del Mayathan (Acuña), y el de Pío Pérez entre otros, igualmente aportan valiosa información no solo sobre los antiguos sistemas clasificatorios de la flora y la fauna sino también sobre las enfermedades que las personas padecían así como las habilidades de los especialistas en curarlas. Es así que en el Calepino estos últimos serían descritos por su capacidad de causar o curar enfermedades indistintamente asomando además frecuentes referencias sobre la estrecha relación que había entre la medicina precolombina maya y los ámbitos de lo religioso y la hechicería. En este sentido, Gubler (322-323) señala que en el sistema mesoamericano de creencias, al no existir una clara separación entre los aspectos benévolos y malévolos de los dioses u otras entidades, estos mismos podían ejercer al mismo tiempo tanto influencias positivas como negativas y un ejemplo de los poderes o atributos que pudieron invocar los especialistas prehispánicos es el caso de Ix Chel, deidad maya de la gestación, los trabajos textiles, de la luna y la medicina, cuyas facultades para curar igualmente podían ser utilizadas para provocar enfermedades o ciertos padecimientos en las personas.

Las fuentes más prolijas en información sobre las antiguas practicas medicinales en el área maya peninsular, son los libros de Chilam Balam, especialmente los de Chan Cah, Ixil, Kaua y $\mathrm{Na}$, así como algunas páginas del códice Pérez, extenso documento compilado por Juan Pío Pérez en el siglo XIX. En general, dichos textos versan sobre los pronósticos y la influencia de los astros en el destino y la salud del hombre con base en distintos remedios herbolarios, purgas y sangrías. Complementándose con otros manuscritos como el de Mena y de Sotuta, cuyas recetas curativas usando plantas fueron incorporadas por Roys en The Ethnobotany of the Maya (1976 [1931]), ya mucho antes, en las Relaciones de Yucatán (1898 y 1900), había sido resaltada la importancia de la farmacopea tradicional maya (Gubler: 323). ${ }^{5}$ Aunque asoma frecuentemente la influencia europea en la mayoría de los textos referidos, escritos principalmente durante el siglo XVIII (Calderón 1981; Caledrón 1982; Barrera-Vázquez 1999; Caso

5. Además del de Tekax que aún no ha sido traducido y del Kaua, del cual existe una traducción al inglés, otro grupo de chilames como el Chumayel, Mani y Tizimin, versan principalmente sobre temas míticos, históricos, religiosos, pronósticos, y katúnicos. 
Barrera), abundan en estos las descripciones y nociones florísticas y faunísticas medicinales que desde entonces, heredadas de sus ancestros, preservan los mayas peninsulares.

\section{Las fuentes}

Todo parece indicar que los distintos libros del Chilam Balam fueron redactados de forma independiente por diferentes sacerdotes quienes, utilizando tal vez un mismo almanaque español, traducirían e intercalarían cada quien, su propia versión del conocimiento médico, histórico y esotérico que poseían (Calderón 1982). ${ }^{6}$ Si bien tales manuscritos han servido de base para elaborar diversas obras sobre la flora medicinal, el estudio de la fauna utilizada con iguales fines, particularmente los artrópodos, no ha tenido la misma atención a pesar de las muchas y variadas interacciones entre estos organismos y las comunidades indígenas mesoamericanas (Gómez et al.). En este sentido, el reciente estudio de Cahuich-Campos (2013) sobre las respectivas traducciones de los manuscritos de Chan Cah, Tekax y Nah (Calderón 1981, Calderón 1982) así como del documento facsimilar del Chilam Balam de Ixil (Caso Barrera), en cuyas recetas médicas figuran diversos artrópodos, permitió identificar las partes y productos de los animales empleados así como las formas de preparación en diversos tratamientos y enfermedades que se atendían poco antes, así como durante la época colonial temprana. Comprendiendo un total de 49 recetas, 21 correspondieron al texto de Ixil, 20 al de Tekax y Nah, y 8 al de Chan Cah, y en conjunto, todas refirieron el uso de 13 tipos de artrópodos para curar 39 padecimientos (Cahuich-Campos). Mientras que 38 de las enfermedades referidas fueron aquellas reconocidas por la ciencia médica occidental, que afectan el cuerpo "físico de la persona", y su causalidad es natural o terrenal, una más, reconocida como el viento o tamcaz en el manuscrito de Chan Cah (Calderón 1982), fue considerada como de filiación cultural. Registrada igualmente en el Calepino de Motul como, Enuaramiento o pasmos, gota coral o enfermedad de frenesí <o epilepsia>, que enmudece, entontece y ensordece al que tiene tamacaz (Ciudad Real: 533), este padecimiento aún es referido hoy día por los mayas peninsulares como iik "aire", "mal aire" o "mal viento", entidad que además de tener la facultad de "traer" a la temible plaga de langosta (Flores 2011: 39), igualmente propiciaba el arribo de seres sobrenaturales tanto del ámbito doméstico como del silvestre, con capacidad de ocasionar toda clase de perjuicios o de provocar enfermedades, sea en personas, animales, plantas u objetos.

6. Incluso se piensa que debieron tener acceso a una Biblia y a diversos textos españoles como el Cantar del Mío Cid, textos relacionados con temas de astronomía y medicina (Caso Barrera). 
En general, los padecimientos referidos no solo aluden a enfermedades naturales sino también a síntomas o malestares que asimismo pudieron ser categorizados de acuerdo a aquellos sistemas y aparatos del cuerpo que afectaban incluyendo además las enfermedades de filiación cultural así como ciertos casos como el de la mosca (Diptera), considerada en el Chilam de Chan Cah, como indicador de enfermedad y muerte (Alves y Rosa, citados por Cahuich-Campos: 18). ${ }^{7}$ Otros males categorizados como indefinidos, y para los que se menciona el uso de artrópodos para tratarlos, fueron el "de las hormigas recias", la fiebre del avispero, la gota, hinchazón de garganta, y dolor de corazón cuyo uso implicaba que los animales fuesen empleados, solos o mezclados con plantas medicinales nativas y/o con productos provenientes de Europa, para atender males relacionados principalmente con los sistemas digestivo, nervioso y urinogenital. ${ }^{8} \mathrm{Tal}$ sincretismo habla no solo de las variadas formas en que los mayas peninsulares irían amalgamando las nuevas concepciones europeas con sus propios conocimientos ancestrales sino también de las maneras en que estos mismos se adaptarían a los complejos procesos históricos a los que se vieron sometidos sin que por ello dejasen de dar cuenta de sus profundos conocimientos naturalistas en dichos manuscritos. Las nociones entomoterapéuticas identificadas en los Chilames incluyeron igualmente el uso de tratamientos basados en principios homeopáticos para atender algunas lesiones y envenenamientos (Caso Barrera ). ${ }^{9}$ Ello concuerda con lo señalado por Ramos-Elorduy, respecto al llamado "principio de similitud" que igualmente fue practicado en muchas otras partes de Mesoamérica para tratar afecciones como por ejemplo cálculos en la vejiga por la semejanza entre este órgano y los femorales de la pata trasera de los chapulines y grillos, entre otros tetigónidos. Bajo la misma hipótesis, el gusano blanco también se usa contra la impotencia sexual, mientras que las cucarachas se emplean contra el cáncer, malestares estomacales, tos y regeneración física.

7. Descrito en el texto como: "arte para ver la orina de los enfermos, así como la de los sanos... Si se mira la orina blancuzca, espesa y que al rociarse en el suelo están mosqueando la orina, está muy enfermo, si no la mosquean no morirá".

8. Las equivalencias entre las categorías de enfermedades registradas en los manuscritos de Chan Cah, Tekax y Nah e Ixil, con respecto a las categorías propuestas por Alves y Rosa, así como el uso múltiple de los artrópodos para atender un mismo padecimiento o enfermedad, puede consultarse en Cahuich-Campos (20-22).

9. Con base en el principio de similitud enunciado por Hahnemann en 1796: "toda sustancia capaz de inducir, a dosis ponderable, en un individuo sano una serie de síntomas patológicos es susceptible, a dosis bajas y especialmente preparadas, de hacer desaparecer los síntomas parecidos en el enfermo que lo presente", lo que se conoce en latín como Similia Similibus Curantur (lo semejante se cura con lo semejante) (Cornillot: 49-50). 
Para el caso del área maya peninsular, el manuscrito de Ixil por ejemplo, contiene recetas para curar la picadura de avispa (Vespidae) utilizando como ingrediente principal el mismo veneno del insecto, o la del alacrán (Centruroides sp), para cuyo ataque se "debía tomar el alacrán que lo pico, rasgar su vientre, sacarle las entrañas y embarrarlas sobre la picadura, con lo que se quitará" (Caso Barrera), y en el caso de la fiebre del avispero, en el chilam de Tekax y Nah se recomienda el uso de la colmena de la avispa (Calderón 1982). Respecto a las picaduras de alacranes (Centruroides ochraceus y C. gracilis), lo anterior se constata hoy día en diversas comunidades de la península donde los arácnidos son utilizados para desvanecer verrugas una vez que estos fueron tostados y pulverizados para luego integrarlos a la comida de la persona afectada..$^{10}$ Aunado al marcado simbolismo que los mayas prehispánicos atribuirían a los alacranes, sea por su relación con la fertilidad de los humanos y de la tierra, o como marcador astronómico y ambiental (Flores 2014), los síntomas producidos por su veneno igualmente fueron registrados en el Códice Madrid (Láminas 79, 80, 82, 83 y 84), donde el personaje que representa a la deidad Ek Chuah o alacrán negro, luce en su boca labios gruesos y rojos lo que podría estar figurando la sensación de su engrosamiento y calor luego de la picadura del arácnido (Sotelo 2012 : 33-35), y muy seguramente, del llamado alacrán negro (Centuroides gracilis), una de las dos especies más comunes en la península de Yucatán (Pinkus et al.: 155); (fig. 3).

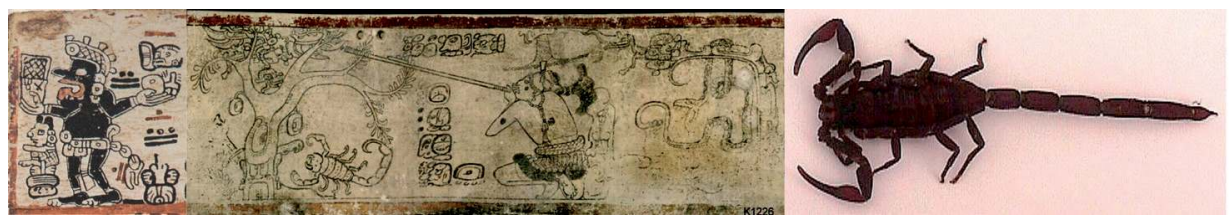

Figura 3. Detalle de la deidad Ek Chuah o alacrán negro, en la lámina $83^{a}$ del códice Madrid; vasija K1226

Aún hoy día, estos espiritistas mayas, cuyas funciones rebasan las concernientes a la atención médica, igualmente dirigen otras ceremonias y rituales relacionados particularmente con las actividades agrícolas y, tal como fuesen registrados a finales del siglo XVII, dichas nociones entomológicas pudieron tener su origen en tiempos remotos como resultado de la transmisión de antiguos conocimientos empíricos sobre estos, y muchos otros tipos de artrópodos (fig. 4).

10. Igualmente, la hormiga león, o ue uech luum (Myrmeleon sp), se emplea en su etapa larvaria para eliminar el pie de atleta colocando el insecto entre los dedos o deshaciéndolo contra la piel agrietada de quien lo padece. 


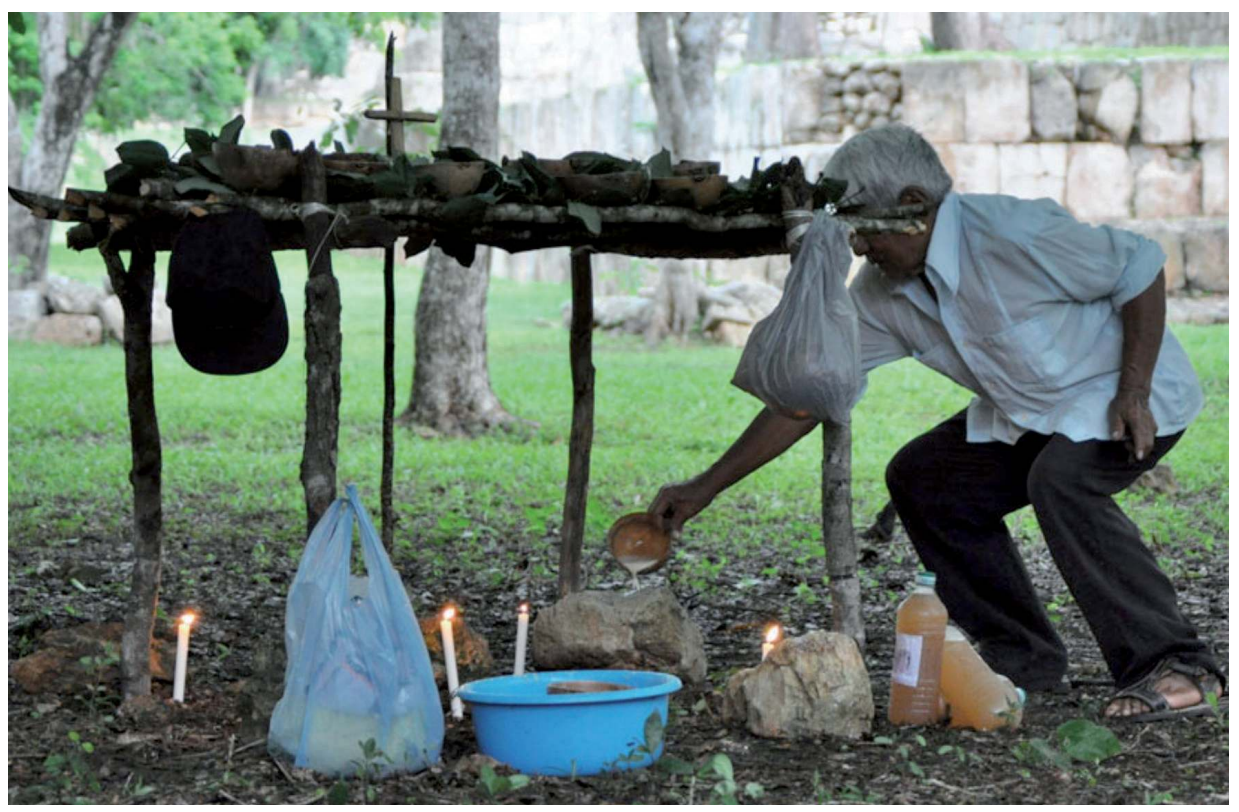

Figura 4. Dionisio Dzib, h-men de Kikil, Yucatán, instala el altar de ofrenda a los Yum tsiles.

Respecto al conocimiento de las partes y/o productos de los artrópodos que debían utilizarse en la elaboración de las recetas en los Chilames, se señala el uso de aceites, entrañas, cuerpo y aguijones así como la cera, miel, veneno, e incluso las larvas de algunos organismos junto con el empleo de panales de abejas y avispas. Hoy día, la utilización de la miel, cera y panales sigue siendo una práctica terapéutica común en diversas comunidades mayas peninsulares para el tratamiento de afecciones oculares o de oídos, como antibiótico para problemas respiratorios, digestivos, en quemaduras o heridas de la piel siendo usados además de distintas formas para atender a las mujeres parturientas (González y Quezada-Euán). Las nociones terapéuticas registradas en los Chilames incluyen igualmente preparaciones en las que se mezclan diversos tipos de artrópodos en una misma receta y con estas se atendían ocho distintos padecimientos o enfermedades (Cahuich-Campos : 16-23). Junto con el tratamiento para la fiebre del "avispero" en el que se utilizaban colmenas de la avispa (Vespidae), para otras afecciones se empleaba la libélula (Odonata: Anispotera) y para evitar la orina se recomendaba el uso de la grana cochinilla (Dactylopius coccus) y el grillo (Orthoptera) en un mismo remedio (Calderón 1981 y 1982); véase síntesis en el cuadro 1). 


\begin{tabular}{|c|c|c|c|c|}
\hline Artrópodo & $\begin{array}{c}\text { Nombre } \\
\text { maya }\end{array}$ & $\begin{array}{l}\text { Nombre } \\
\text { científico }\end{array}$ & $\begin{array}{c}\text { Partes o } \\
\text { productos } \\
\text { empleados }\end{array}$ & Enfermedad / Padecimiento \\
\hline \multirow[t]{3}{*}{ Abeja } & Kaab & Melipona sp & Cera & $\begin{array}{l}\text { Paño en la cara, calentura por calor, corrupción de la } \\
\text { sangre, diarrea, dolores de cabeza, de corazón, de } \\
\text { oído, de vientre pulsante, evacuaciones del estómago, } \\
\text { flatulencia, hinchazón garganta, insanos pares retiene la } \\
\text { mujer, lombrices, malestar en ojos, mordedura víbora } \\
\text { o perro rabioso, no orinar, orinar materia, paludismo, } \\
\text { placenta retenida, tisis y tosferina, vómito de sangre, } \\
\text { piixtola y viento tamcaz (ambas enfermedades } \\
\text { culturales) }\end{array}$ \\
\hline & & & Panal y miel & Viruela negra (Ekpeo) \\
\hline & & & Aceite & Diarrea \\
\hline Alacrán & Slina'an & Centruroides $s p$ & Entrañas & Picadura de alacrán \\
\hline $\begin{array}{l}\text { Alacrán o } \\
\text { araña látigo }\end{array}$ & Ch'íintun & $\begin{array}{l}\text { Amblypygi } \\
\text { paraphrynus }\end{array}$ & Cuerpo & Insolación en niños \\
\hline \multirow[t]{9}{*}{ Avispa } & Xuux & Vespidae & Panal & Fiebre del "avispero" \\
\hline & & & $\begin{array}{l}\text { Panal (sin } \\
\text { animales) }\end{array}$ & Orinar materia \\
\hline & & & Cuerpo y panal & Hinchazón rodillas \\
\hline & & & Panal & Hinchazón rodillas y articulaciones \\
\hline & & & Veneno & Picadura de avispa \\
\hline & & & Cuerpo & No orinar \\
\hline & Xpéet k'ïnil & & panal & Fiebre del "avispero" \\
\hline & Bo'bo'ote' & & cuerpo & Bobante kak \\
\hline & Xuux & & Cuerpo y panal & Hinchazón rodillas \\
\hline $\begin{array}{l}\text { Mosca de } \\
\text { miel }\end{array}$ & Xiik' & $\begin{array}{l}\text { Apidae: } \\
\text { Meliponini }\end{array}$ & Larvas y panal & Trabazón de los pares en la mujer \\
\hline $\begin{array}{l}\text { Comején } \\
\text { (termita) }\end{array}$ & Kamaz & Isoptera & Aguijón & Dolor de rodilla \\
\hline $\begin{array}{l}\text { Grana } \\
\text { cochinilla }\end{array}$ & Mukay & $\begin{array}{l}\text { Dactylopius } \\
\text { coccus }\end{array}$ & Cuerpo & No orinar \\
\hline Grillo & Máas & Orthoptera & Cuerpo & No orinar \\
\hline $\begin{array}{l}\text { Hormiga } \\
\text { trepadora }\end{array}$ & Zinic & Formicidae & Larvas & Nube en los ojos \\
\hline Kan kub & Kan kub & & Larvas & Sangre de mujer \\
\hline Langosta & Sáak & $\begin{array}{l}\text { Schistocerca } \\
\text { piceifrons }\end{array}$ & Cuerpo & Hipo \\
\hline \multirow[t]{2}{*}{ Libélula } & Tulix & $\begin{array}{l}\text { Odonata: } \\
\text { Anisoptera }\end{array}$ & Larvas & Fiebre del "avispero" \\
\hline & & & Cuerpo & Escupir sangre (no vómito de sangre) \\
\hline Mosca & Ya'axkach & Díptera & $\begin{array}{l}\text { Indicador de } \\
\text { enfermedad y } \\
\text { muerte }\end{array}$ & Ver orina de enfermos \\
\hline
\end{tabular}

Cuadro 1. Artrópodos con uso medicinal, partes o productos empleados y enfermedades o padecimientos mencionados en los manuscritos coloniales mayas (reelaborado de Cahuich-Campos: 19-20).

\section{Nota final}

Como parte de un trabajo en curso más amplio, el examen de muy diversas fuentes de información pone de manifiesto el amplio conocimiento empírico y la importancia cultural que distintos tipos de animales artrópodos tuvieron, y aún hoy día tienen, en la medicina tradicional de los pueblos mayas peninsu- 
lares. Habiendo sido transmitidas de generación en generación, son no pocas las nociones entomológicas vigentes aún hoy día mismas que aparecen en distintas prácticas terapéuticas tradicionales, aspecto que destaca no solo como uno de los rasgos distintivos de la cultura maya en la península de Yucatán, sino también como elemento clave de sus propias estrategias de resilencia socioambiental basado en gran medida en el uso múltiple de los recursos y la naturaleza (Toledo et al.). Los resultados obtenidos hasta el momento abren la posibilidad de ampliar y diversificar el estudio de las propiedades medicinales de los artrópodos, y sobre todo aquellos que fueron empleados desde la época prehispánica. A pesar de las dificultades intrínsecas del estudio arqueológico de las antiguas practicas de sanación, los datos etnográficos del área mesoamericana representan sólidas plataformas para indagar sobre aspectos particulares de la entomoterapia como, por ejemplo, la aplicación de los piquetes de las avispas para tratar la histeria, menopausia y síntomas neurológicos. Además del extendido uso alimenticio de los grillos en Oaxaca, al sur de México, quedan también por explorar otras formas de aprovechamiento tanto de cucarachas como abejorros, insectos utilizados de broncodilatadores para el dolor o inflamación de la garganta así como antibióticos. Siendo animales muy comunes por toda la región neotropical, distintos tipos de hormigas representan igualmente otros temas de estudio particularmente interesantes en tanto su utilización para activar el sistema inmunológico humano así como por las propiedades antibióticas que algunas especies secretan y que sirven para curar heridas.

\section{Obras y estudios citados}

ACUÑA René. 1993, Bocabulario del Mayathan, México, Centro de Estudios Mayas, Instituto de Investigaciones Filológicas, UNAM, col. «Fuentes para el estudio de la cultura maya, $\mathrm{n}^{\circ} 10 », 666 \mathrm{p}$.

ALVES RÓMULO R.N. y ROSA I.L. 2006, « From Cnidarians to Mammals: The Use of Animals as Remedies in Fishing Communities in NE Brazil », Journal of Ethnopharmacology 107: 259-276.

ALVES RÓMULO R.N y ALVES H. N. 2011, « The Faunal Drugstore: Animal-Based Remedies Used in Traditional Medicines in Latin America Brazil ", Journal of Ethnobiology and Enthnomedicine 7: 1-43.

ARZÁPALO Marín Ramón. 1987, El ritual de los Bacabes, México, UNAM, $197 \mathrm{p}$.

BARRERA-VÁZQUEZ Alfredo et al. 1991 [1980], Diccionario maya, Maya-español, Español-maya, México, Porrúa.

BARRERA-VÁZQUEZ Alfredo. 1999, «Las fuentes para el estudio de la medicina nativa de Yucatán », Revista Biomédica 10: 253-261. 
BRACAMONTE y SOSA Pedro. 2010, Tiempo cíclico y vaticinios: ensayos etnohistóricos sobre el pensamiento maya, México, CIESAS y Editorial Porrúa, $235 \mathrm{p}$.

BRUSCA, R. C. y BRUSCA, G. J. 1990, Invertebrates, Sunderland (MA), Sinauer Associates, $979 \mathrm{p}$.

CAHUICH-CAMPOS Diana. 2013, "Los artrópodos utilizados en la medicina tradicional maya mencionados en los libros de Chilam Balam de Chan Cah, Tekax y Nah e Ixil », Etnobiología 11 (2): 16-23.

CALDERÓN M. Héctor. 1981, Manuscritos de Tekax y Nah, México, Grupo Editorial Dzibil.

. 1982, Manuscritos de Chan Cah, México, Grupo Editorial Dzibil.

CASO BARRERA Laura. 2011, Chilam Balam de Ixil. Facsimilar y estudio de un libro maya inédito. México, CONACULTA-INAH (Consejo Nacional para la Cultura y las Artes - Instituto Nacional de Antropología e Historia), 283 p.

CIUDAD REAL Antonio. 2001, Calepino Maya de Motul. México, Plaza y Valdés Editores, Edición crítica y anotada por René Acuña, 597 p.

CORNILlOT P. 2002, Tratado de Homeopatía, Barcelona, Editorial Paidotribo, $676 \mathrm{p}$.

COSTA-NETO Eraldo. 2005, "Animal-Based Medicines: Biological Prospection and the Sustainable Use of Zootherapeutic Resources ", Anais da Academia Brasileira de Ciências 77 (1): 33-43.

COSTA-NETO E., RAMOS ELORDUY Julieta y PINO J. M. 2006, « Los insectos medicinales de Brasil: Primeros resultados ", Boletín Sociedad Entomológica Aragonesa 38: 395-414.

DE LA FUENTE Freyre J.A. 1994, Zoología de Artrópodos, Madrid, McGraw-Hill/Interamericana.

FLORES, Fabio. 2011, "Las plagas de langosta en el área maya: ambiente e historia de una antigua calamidad ", Península 6 (2): 27-46.

. 2014, «Nociones entomológicas de los antiguos pueblos mayas ", in Memorias del IX Congreso Nacional de Etnobiología, San Cristóbal de las Casas, Chiapas, México (en publicación).

GÓMEZ Á. G., REYES G. S. R., TEUTLI S. C. y VALADÉZ A. R. 2007, « La medicina tradicional prehispánica, vertebrados terrestres y productos medicinales de tres mercados del Valle de México », Etnobiología 5: 86-98.

GONZÁLEZ ACERETO Jorge Ángel A. y QUEZADA-EUÁN José J. 2010, "Producción tradicional de miel: abejas nativas sin aguijón (trigonas y meliponas). » in R. Durán y M. Méndez (eds.), Biodiversidad y desarrollo humano en Yucatán, Mérida, CICY/PPD-FMAM/Conabio/Seduma, 496 p.

GUBLER Ruth. 2001, « La medicina tradicional en Yucatán a través de los siglos » in Yucatán a través de los siglos, Memorias del $49^{\circ}$ Congreso Internacional 
de Americanistas (Quito), Mérida, Ediciones de la Universidad Autónoma de Yucatán, 229-248.

HART, Thomas. 2008, The Ancient Spirituality of the Modern Maya, Albuquerque, University of New Mexico Press, $270 \mathrm{p}$.

HERRERA y TORDESILLAS Antonio de. 1726-30, Historia General de los Hechos de los Castellanos en las Islas y Tierra Firme del Mar Océano, Madrid, La oficina real de Nicolás Rodríguez Franco, con privilegio de su Majestad.

LANDA FRAY Diego de. 1986, Relación de las Cosas de Yucatán, edición de A.M. Garibay, México, Editorial Porrúa.

MARCUS Joyce. 1982, "The Plant World of the Sixteenth and Seventeenth Century Lowland Maya » in Kent V. Flannery (ed.), Maya Subsistence: Studies in Memory of Dennis E. Puleston, New York, Academic Press, 239-273.

PENICHE Paola. 2010, Tiempos aciagos: las calamidades y el cambio social del siglo XVIII entre los mayas de Yucatán, México, México, CIESAS y Editorial Porrúa, 359 p.

PÉREZ SUÁREZ Tomás. "Las lenguas mayas: historia y diversidad ». Revista Digital Universitaria [en línea]. 10 de agosto de 2004, Vol. 5, No. 7. [Consultada: agosto 2013]. Disponible en Internet:http://www.revista.unam.mx/vol.5/ num7/art45/art45.htm.

PÉREZ Juan Pío et al. 1877, Diccionario de la lengua maya, Mérida, Editorial J.F. Molina Solís, 437 p.

PINKUS-RENDÓN M.A. et al. 1999, "Alacranes sinantrópicos de Mérida, Yucatán, México », Revista Biomédica 10:153-158.

RAMOS ELORDUY J. y J. M. PINO M. 1988, " The Utilization of Insect in the Empirical Medicine of the Ancient Mexicans ", Journal of Ethnobiology 8: 61-72.

ROYS Ralph L. 1976 [1931], The Ethnobotany of the Maya, Philadelphia, Institute for the Study of Human Issues.

SERRANO-GONZÁLEZ Rafael et. al. 2013, "Los artrópodos medicinales en tres fuentes novohispanas del siglo XVI », Etnobiología 11 (2), 24-34.

SCHELE Linda y FREIDEL David. 1990, A forest of Kings. Untold Stories of the Ancient Maya, New York, William Morrow and Co. 554 p.

SCHLESINGER Victoria. 2001, Animals and Plants of the Ancient Maya, Austin, University of Texas Press, 373 p.

. 2012, «El arte de medir el tiempo », Artes de México 107: 14-17.

TOLEDO M. V., BARRERA-BASSOLS, N., GARCÍA, F. E. y ALARCÓN-CHAIRES P. 2008, « Uso múltiple y biodiversidad entre los mayas yucatecos (México) », Interciencia 33: 345-352. 
Resumen: El estudio de distintas recetas, formulas adivinatorias y augurios registrados en algunas fuentes etnohistóricas y relacionadas con el uso de diversos tipos de artrópodos permitió aproximarse a las antiguas prácticas médicas tradicionales así como a la antigua cosmovisión maya. Además de aportar información sobre los tipos de padecimientos y sus formas de sanación, el examen de dichas prácticas permitió conocer ciertas relaciones de la etología y biología de los animales empleados con ciclos naturales y eventos climatológicos así como algunas de las ancestrales formas en que estos pudieron ser percibidos, catalogados y aprovechados en la época prehispánica. La información obtenida, que incluye datos sobre las partes del animal usadas, su preparación y la identificación de más de 20 artrópodos utilizados tanto para curar enfermedades como para la adivinación, constata el amplio conocimiento empírico y la importancia de este tipo de organismos entre los pueblos mayas peninsulares.

Palabras clave: entomoterapia, medicina tradicional maya, adivinación

Résumé : L'étude de diverses recettes, formules divinatoires et prédictions, recueillies dans certaines sources ethno-historiques, et liées à l'usage de différents types d'arthropodes, contribue à la compréhension des anciennes pratiques médicales traditionnelles et à celle de l'ancienne cosmovision maya. En plus de fournir des données sur les types de pathologies et les procédés thérapeutiques, l'examen de ces pratiques aide à éclaircir certaines relations entre l'éthologie et la biologie des animaux utilisés, d'une part, et les cycles naturels et événements climatologiques, d'autre part, ainsi qu'à faire apparaître, à certains égards, de quelles façons ces animaux étaient perçus, catalogués et utilisés à l'époque préhispanique. Les données obtenues, qui incluent des renseignements sur les parties des animaux utilisées, les préparations de celles-ci et l'identification de plus de 20 arthropodes servant tant à traiter des maladies qu'à la divination, permettent de faire état de l'ampleur des connaissances empiriques et de l'importance de ce type d'organismes pour les peuples mayas de la Péninsule du Yucatán.

Mots-clés : entomo-thérapie, médecine traditionnelle maya, divination

Diana Cahuich-Campos es bióloga por la Universidad Nacional Autónoma de México (UNAM) con doctorado en Ecología y Desarrollo Sustentable por El Colegio de la Frontera Sur (ECOSUR). Es miembro de la Red de Antropología e Historia de la Diversidad Cultural y Biológica del Sureste Mexicano (AHDIVERSUR). Sus temas de interés son el huerto familiar y su agrobiodiversidad como patrimonio cultural y biológico, así como su función en las prácticas medicinales y rituales de las familias campesinas mayas en Campeche, temas en los que tiene diversas publicaciones. Actualmente realiza una estancia postdoctoral en ECOSUR en un proyecto en colaboración con la Universidad de Massachusetts.

Diana Cahuich-Campos est biologiste, diplômée de I'Université Nationale Autonome du Mexique (UNAM) et a un doctorat en Ecologie et Développement Durable obtenu au Collège de la Frontière Sud (ECOSUR) à San Cristobal de las Casas, Chiapas. Elle est membre du Réseau d'Anthropologie et d'Histoire de la Diversité Culturelle et Biologique du Sud-est Mexicain (AHDIVERSUR). Ses recherches portent sur le potager familial et son agro-diversité comme patrimoine culturel et biologique, ainsi que sur sa fonction dans les pratiques médicales et rituelles des familles paysannes mayas de l'Etat de Campeche, des thèmes dont elle a traité dans diverses publications. Actuellement, elle fait un post-doctorat à ECOSUR dans le cadre d'un projet mené en collaboration avec l'Université du Massachusetts.

Fabio Flores Granados es biólogo por la Universidad Autónoma Metropolitana (UAM) con doctorado en Antropología por el Instituto de Investigaciones Antropológicas de la Universidad Nacional Autónoma de México (UNAM). Sus temas de interés son las formas de percepción y uso de los recursos faunísticos en la época prehispánica, tópicos de los que tiene diversas publicaciones. Es Investigador del Centro Peninsular en Humanidades y en Ciencias Sociales (CEPHCIS) de la UNAM, en Mérida, Yucatán. Es miembro de la red CONACYT (Consejo Nacional de Ciencia y Tecnología) de Etnobiología 
y Patrimonio Biocultural y forma parte de los Comités Directivos de la Asociación Etnobiológica Mexicana (AEM) y de la Sociedad Latinoamericana de Etnobiología (SOLAE).

Fabio Flores Granados est biologiste, diplômé de I'Université Autonome Métropolitaine (UAM, Mexico) et a un doctorat en Anthropologie obtenu à I'Institut de Recherches Anthropologiques de I'Université Nationale Autonome du Mexique (UNAM). Ses recherches portent sur l'usage et la manière de se représenter les ressources faunistiques à l'époque préhispanique ; elles ont fait l'objet de diverses publications. Fabio Flores est chercheur au Centre Péninsulaire en Humanités et Sciences Sociales (CEPHCIS) de I'UNAM, à Mérida (Yucatan). Il est membre du réseau CONACYT (équivalent du CNRS au Mexique) sur l'Ethnobiologie et le Patrimoine Bioculturel. Il fait également partie du comité de direction de l'Association d'Ethnobiologie Mexicaine (AEM) et de celui de la Société Latino-américaine d'Ethnobiologie (SOLAE). 